\title{
Unsteady Natural Convection Flow past an Infinite Cylinder with Thermal and Mass Stratification
}

\author{
Ashish Paul ${ }^{1}$ and Rudra Kanta Deka ${ }^{2}$ \\ ${ }^{1}$ Department of Mathematics, Cotton College State University, Guwahati 781001, India \\ ${ }^{2}$ Department of Mathematics, Gauhati University, Guwahati 781014, India \\ Correspondence should be addressed to Ashish Paul; ashpaul85@gmail.com
}

Received 29 June 2016; Revised 8 November 2016; Accepted 6 December 2016; Published 11 January 2017

Academic Editor: Alberto Cardona

Copyright ( 2017 A. Paul and R. K. Deka. This is an open access article distributed under the Creative Commons Attribution License, which permits unrestricted use, distribution, and reproduction in any medium, provided the original work is properly cited.

This paper presents an analytical solution of unsteady one-dimensional free convection flow past an infinite vertical circular cylinder in a stratified fluid medium. The dimensionless coupled linear governing partial differential equations are solved by Laplace transform technique for unit Prandtl number and Schmidt number. Effects of various physical parameters are presented with graphs. Numerical values of boundary layer thickness for different parameters are presented in table. Due to the effects of thermal and mass stratifications, the velocity, temperature, and skin friction, Nusselt number shows oscillatory behaviour at smaller times and then reaches steady state at larger times.

\section{Introduction}

Natural convection flows with heat and mass stratification are frequently encountered in nature. These types of problem over vertical cylinder have wide range of applications in the field of science and technology such as startup of chemical reactors and emergency cooling of nuclear fuel elements. In glass and polymer industries, hot filaments are considered as vertical cylinder and cooled as they pass through the surrounding environment. Free convective flows driven by temperature and concentration difference have been studied extensively. When both the temperature and concentration differences occur simultaneously, the free convective flow can become quite complex.

Gebhart and Pera [1] analysed the steady combined buoyancy effects on vertical natural convection flows. Bottemanne [2] studied the combined effect of heat and mass transfer in the steady laminar boundary layer of a vertical cylinder placed in still air for $\operatorname{Pr}=0.71$ and $\mathrm{Sc}=0.63$. Chen and Yuh [3] presented a numerical study of steady heat and mass transfer processes near cylinder with uniform wall heat and mass fluxes and wall temperature. Their study covered a wide range of radii and Prandtl numbers. Velusamy and Garg [4] studied natural convection adjacent to a heat generating vertical cylinder. Ganesan and Rani [5] presented a numerical solution for the transient natural convection flow over a vertical cylinder under the combined buoyancy effect of heat and mass transfer. Numerical analysis of two-dimensional unsteady natural convective flow past semi-infinite vertical cylinder with heat and mass transfer under different physical situations was studied by Ganesan and Loganathan [6-8]. Rani [9] presented a numerical study on transient natural convection along vertical cylinder with variable surface temperature and mass diffusion. Devakar et al. [10] presented closed form solution for Poiseuille flow, Couette flow, and generalized Couette flows of an incompressible couple stress fluid between two concentric circular cylinders with slip boundary condition. Recently, Deka et al. [11] presented the analytical investigation of one-dimensional unsteady natural convection flow past an infinite vertical cylinder with heat and mass transfer under the effect of constant heat flux at the surface of the cylinder. They have shown that the velocity and temperature increase unboundedly with time, while the concentration approaches steady state at larger times.

In recent times many researchers have shown interests in the study of stratification effects on transient natural convective flows along vertical bodies under various physical situations. Takhar et al. [12] presented a numerical study of 
natural convection boundary layer flow over a continuously moving vertical surface immersed in a thermally stratified medium by an implicit finite difference scheme. Again, Takhar et al. [13] investigated the natural convection flow past a vertical cylinder embedded in a thermally stratified highporosity medium. They solved the coupled nonlinear partial differential equations by finite difference as well as perturbation technique and found that separation of flow occurs for some values of stratification parameter. Loganathan and Ganesan [14] presented a numerical study of free convective flow of a viscous incompressible fluid past a moving, semiinfinite vertical cylinder with constant temperature and mass diffusion in a thermally stratified medium by employing a finite difference scheme of Crank-Nicolson type.

Shapiro and Fedorovish [15] presented analytical solution of one-dimensional laminar natural convection along an infinite vertical plate by introducing the pressure work term and the ambient thermal stratification in the thermodynamic energy equation for the case of unit Prandtl number. They have shown that thermal stratification provides a negative feedback mechanism: warm fluid rises, expands, and cools relative to the environment, whereas cool fluid subsides, compresses, and warms relative to the environments. Later on, Shapiro and Fedorovich [16] carried out study on natural convection in a stably stratified fluid along vertical plates and circular cylinders seeking solutions in the form of harmonic oscillators.

In recent times, the effect of double stratifications, namely, thermal stratification and mass stratification, has been considered by different researchers. For example, Cheng [17] studied the coupled heat and mass transfer by natural convection near a vertical wavy surface in a non-Newtonian fluid saturated porous medium with thermal and mass stratification and obtained solutions by collocation method. Recently, Srinivasacharya and Reddy [18], Srinivasacharya and RamReddy [19], Rathish Kumar and Krishna Murthy [20], and Neagu [21] have investigated numerically on flow past plates and wavy surfaces taking double stratifications into account.

Deka and Paul $[22,23]$ presented the analytical investigation of transient free convection flow past an infinite moving vertical cylinder in a stably stratified fluid including thermal stratification by employing Laplace transform technique. Very recently Deka and Paul [24] presented analytical investigation to study the effects of thermal stratification and mass stratification on natural convection heat and mass transfer over moving vertical cylinder. This motivates undertaking this study. This paper presents an analytical investigation of one-dimensional free convective flow past a stationary infinite vertical cylinder with combined effects of thermal and mass stratification. The unsteady nondimensional governing linear equations are solved by Laplace transform technique for the case of unit Prandtl number and unit Schmidt number. Solutions are presented in closed form and this is always necessary for validating numerical models. Also solutions of unsteady state for larger time are compared with the solutions of steady state.

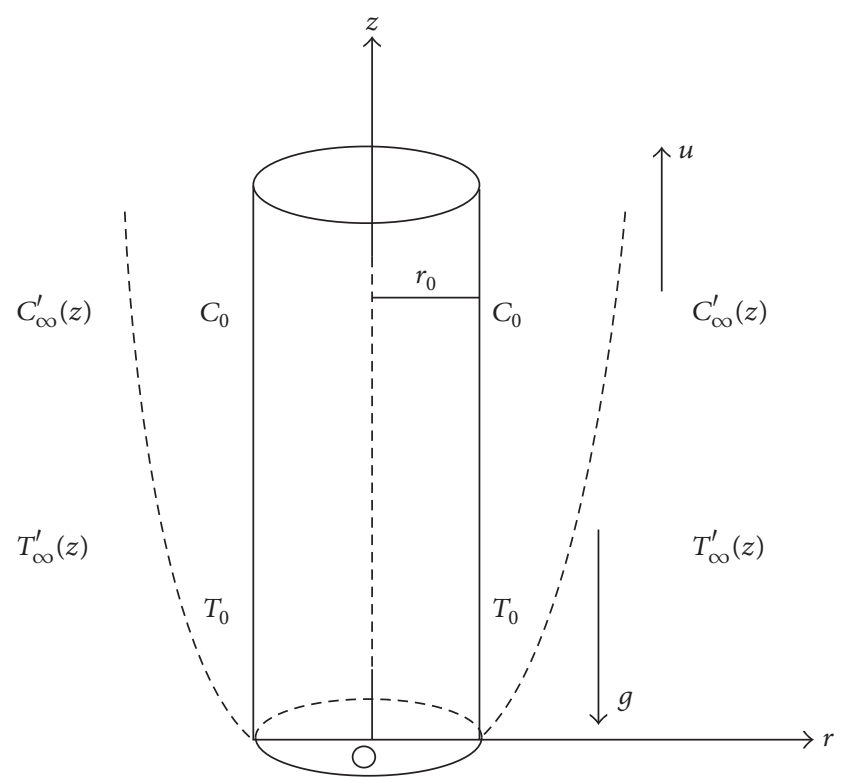

FIGURE 1: Sketch of the geometry.

\section{Mathematical Analysis}

Consider an unsteady, laminar, and incompressible viscous flow past an infinite vertical cylinder of radius $r_{0}$ with constant temperature and concentration in presence of thermal and mass stratification. The $z$-axis of the cylinder is taken vertically upward along the axis of the cylinder and the radial coordinate $r$ is taken normal to the cylinder as shown in Figure 1. The physical model and coordinate system of the flow problem is shown in Figure 1. Upon commencement of the transient, we consider the fluid moving up from the leading edge $(z=0)$ parallel to the cylinder as a wave, in front of which the velocity, temperature, and concentration are only functions of the time and the radial distance $r$ from the cylinder. Behind the wave there must be a dependence on the vertical coordinate, $z$. The basic premise in this work is that convective effects will begin at a position, $z$, as soon as fluid which was initially located at the leading edge rises to this position, regardless of the distance, $r$, away from the cylinder at which it first arrives. Since the surface temperature and concentration above the leading edge are uniform with $z$, the temperature of the fluid and concentration may be assumed to be independent of $z$. In addition, the vertical velocity, $u$, must be independent of $z$ and from the continuity equation, the velocity normal to the plate is seen to be zero, except that the temperature and concentration of the ambient fluid are function of the vertical distance $z$ only. At time $t^{\prime}>0$, the uniform temperature $\left(T_{0}\right)$ and concentration $\left(C_{0}\right)$ are specified at the surface of the cylinder. Viscous dissipation terms have been neglected. All derivatives in the direction parallel to the cylinder are zero, except $d T_{\infty}^{\prime} / d z$ and $d C_{\infty}^{\prime} / d z$ termed as thermal stratification and mass stratification, respectively. Here, $T_{\infty}^{\prime}$ and $C_{\infty}^{\prime}$ are the temperature and concentration of the undisturbed fluid. It is to be noted that initially the fluid may not be stratified, 
but upon commencement of the transient the fluid gets selfstratifications. Then following Boussinesq's approximation, the one-dimensional equations for momentum, energy, and concentration are as follows:

$$
\begin{aligned}
\frac{\partial u}{\partial t^{\prime}}= & \frac{\nu}{r} \frac{\partial}{\partial r}\left(r \frac{\partial u}{\partial r}\right)+g \beta\left(T^{\prime}-T_{\infty}^{\prime}\right) \\
& +g \beta^{*}\left(C^{\prime}-C_{\infty}^{\prime}\right), \\
\frac{\partial T^{\prime}}{\partial t^{\prime}}= & \frac{\alpha}{r} \frac{\partial}{\partial r}\left(r \frac{\partial T^{\prime}}{\partial r}\right)-\gamma u, \\
\frac{\partial C^{\prime}}{\partial t^{\prime}}= & \frac{D}{r} \frac{\partial}{\partial r}\left(r \frac{\partial C^{\prime}}{\partial r}\right)-\xi u
\end{aligned}
$$

with initial and boundary conditions as

$$
\begin{aligned}
& t^{\prime} \leq 0: \quad u=0, \\
& T^{\prime}=T_{\infty}^{\prime} \\
& C^{\prime}=C_{\infty}^{\prime} \\
& \forall r \\
& t^{\prime}>0: \quad u=0, \\
& T^{\prime}=T_{0} \text {, } \\
& C^{\prime}=C_{0} \\
& \text { at } r=r_{0} \\
& u \longrightarrow 0 \text {, } \\
& T^{\prime} \longrightarrow T_{\infty}^{\prime}, \\
& C^{\prime} \longrightarrow T_{\infty}^{\prime}
\end{aligned}
$$

as $r \longrightarrow \infty$.

The physical variables involved above are mentioned in Nomenclature. However, the particular variable $\gamma$, the main concern in our study, is the ambient thermal stratification parameter $\left[\equiv d T_{\infty}^{\prime}(z) / d z\right.$ for Boussinesq flow of liquids or gases, $\equiv d T_{\infty}^{\prime}(z) / d z+g / C_{p}$ for a perfect gas with pressure work term retained], and $z$ is height. Thus $\gamma$ is the combination of thermal stratification and compression, where $d T_{\infty}^{\prime}(z) / d z$ stands for thermal stratification and $g / C_{p}$ for compression. In an adiabatic environment, $d T_{\infty}^{\prime}(z) / d z+$ $g / C_{p}=0$. In that case $d T_{\infty}^{\prime}(z) / d z=-g / C_{p}$ and $\left(-g / C_{p}\right)$ is called the adiabatic temperature gradient and is the largest rate at which the temperature can decrease with height without causing instability. The stability of the atmosphere is determined according to $\gamma>0$ (stable), $\gamma=0$ (neutral), and $\gamma<0$ (unstable). It should be noted, however, that the compression work term is generally quite small (since $g=$ $9.8 \mathrm{~m} \mathrm{~s}^{-2}, C_{p}=1004 \mathrm{~J} \mathrm{~kg}^{-1} \mathrm{~K}^{-1}$ ) and that the main interest in our solutions will probably be in the effects of temperature stratification. We have retained the compression work term so that numerical convection models can be developed for examining the validity of the solution. Also, it can be predicted that the compression, being the additive one to thermal stratification, also plays an important role in the solution of temperature and vertical velocity as well. A similar variable $\xi=d C_{\infty}^{\prime}(z) / d z$ is termed as mass stratification. We shall also study the simultaneous effect of mass stratification in addition to thermal stratification.

Introducing the nondimensional quantities

$$
\begin{aligned}
& R=\frac{r}{r_{0}}, \\
& U=\frac{u r_{0}}{v}, \\
& t=\frac{t^{\prime} v}{r_{0}^{2}} \\
& \theta=\frac{T^{\prime}-T_{\infty}^{\prime}(z)}{T_{0}-T_{\infty}^{\prime}(z)} \\
& \phi=\frac{C^{\prime}-C_{\infty}^{\prime}(z)}{C_{0}-C_{\infty}^{\prime}(z)}, \\
& \operatorname{Pr}=\frac{\nu}{\alpha} \text {, } \\
& \mathrm{Sc}=\frac{\nu}{D} \\
& \mathrm{Gr}=\frac{g \beta r_{0}^{3}\left\{T_{0}-T_{\infty}^{\prime}(z)\right\}}{v^{2}}, \\
& \mathrm{Gc}=\frac{g \beta^{*} r_{0}^{3}\left\{C_{0}-C_{\infty}^{\prime}(z)\right\}}{v^{2}}, \\
& S=\frac{\gamma r_{0}}{T_{0}-T_{\infty}^{\prime}(z)}, \\
& K=\frac{\xi r_{0}}{C_{0}-C_{\infty}^{\prime}(z)}
\end{aligned}
$$

the governing equations (1) reduce to

$$
\begin{aligned}
& \frac{\partial U}{\partial t}=\frac{1}{R} \frac{\partial}{\partial R}\left(R \frac{\partial U}{\partial R}\right)+\operatorname{Gr} \theta+\operatorname{Gc} \phi, \\
& \frac{\partial \theta}{\partial t}=\frac{1}{\operatorname{Pr}} \frac{1}{R} \frac{\partial}{\partial R}\left(R \frac{\partial \theta}{\partial R}\right)-S U, \\
& \frac{\partial \phi}{\partial t}=\frac{1}{\operatorname{Sc}} \frac{1}{R} \frac{\partial}{\partial R}\left(R \frac{\partial \phi}{\partial R}\right)-K U
\end{aligned}
$$

and the corresponding initial and boundary conditions in nondimensional form are

$$
\begin{aligned}
t \leq 0: \quad U & =0, \\
& =0, \\
\phi & =0 \\
t>0: \quad U & =0, \quad \forall R \\
\theta & =1, \\
\phi & =1 \\
U & \longrightarrow 0, \quad \text { at } R=1 \\
\theta \longrightarrow 0, & \\
\phi \longrightarrow 0 & \quad \text { as } R \longrightarrow \infty .
\end{aligned}
$$




\section{Solution Technique}

To solve the governing nondimensional unsteady equations (4) subject to initial and boundary conditions (5), we apply Laplace transform technique for the case of unit Prandtl number and Schmidt number, as for arbitrary Prandtl number or Schmidt number, the Laplace transform technique leads to a difficult inverse transformation step (integrand of the Bromwich integral becomes a complicated multivalued function). Laplace transforms of (4) with initial conditions of (5) with $\operatorname{Pr}=\mathrm{Sc}=1$ become

$$
\begin{aligned}
\frac{d^{2} \bar{U}}{d R^{2}}+\frac{1}{R} & \frac{d \bar{U}}{d R}-p \bar{U}+\operatorname{Gr} \bar{\theta}+\operatorname{Gc} \bar{\phi}=0 \\
\frac{d^{2} \bar{\theta}}{d R^{2}}+\frac{1}{R} \frac{d \bar{\theta}}{d R}-p \bar{\theta}-S \bar{U} & =0 \\
\frac{d^{2} \bar{\phi}}{d R^{2}}+\frac{1}{R} \frac{d \bar{\phi}}{d R}-p \bar{\phi}-K \bar{U} & =0
\end{aligned}
$$

where $p$ is the parameter of the Laplace transformation.

Solutions of (6), (7), and (8) subject to the boundary conditions (5) are obtained as (the processes of determining $\bar{U}, \bar{\theta}$, and $\bar{\phi}$ are presented in Appendix)

$$
\begin{aligned}
\bar{U} & =\frac{\mathrm{Gr}+\mathrm{Gc}}{2 i L}\left\{\frac{K_{0}(R \sqrt{p-i L})}{p K_{0}(\sqrt{p-i L})}-\frac{K_{0}(R \sqrt{p+i L})}{p K_{0}(\sqrt{p+i L})}\right\}, \\
\bar{\theta}= & \frac{\mathrm{Gc}(K-S)}{L^{2}} \frac{K_{0}(R \sqrt{p})}{p K_{0}(\sqrt{p})} \\
& +\frac{S(\mathrm{Gr}+\mathrm{Gc})}{2 L^{2}}\left\{\frac{K_{0}(R \sqrt{p-i L})}{p K_{0}(\sqrt{p-i L})}\right. \\
& \left.+\frac{K_{0}(R \sqrt{p+i L})}{p K_{0}(\sqrt{p+i L})}\right\}, \\
\bar{\phi} & =\frac{\mathrm{Gr}(S-K)}{L^{2}} \frac{K_{0}(R \sqrt{p})}{p K_{0}(\sqrt{p})} \\
& +\frac{K(\mathrm{Gr}+\mathrm{Gc})}{2 L^{2}}\left\{\frac{K_{0}(R \sqrt{p-i L})}{p K_{0}(\sqrt{p-i L})}\right. \\
& \left.+\frac{K_{0}(R \sqrt{p+i L})}{p K_{0}(\sqrt{p+i L})}\right\} .
\end{aligned}
$$

Inverse Laplace transforms of (9) give the expressions of velocity, temperature, and concentration profile [following Deka and Paul [24], Carslaw and Jaeger [25]] as follows:

$$
\begin{aligned}
U & =\frac{\mathrm{Gr}+\mathrm{Gc}}{2 L i}\left\{\frac{K_{0}(R \sqrt{-i L})}{K_{0}(\sqrt{-i L})}-\frac{K_{0}(R \sqrt{i L})}{K_{0}(\sqrt{i L})}\right\} \\
& +\frac{2(\mathrm{Gr}+\mathrm{Gc})}{\pi L}
\end{aligned}
$$

$$
\begin{aligned}
& \cdot \int_{0}^{\infty}\left\{\frac{e^{-V^{2} t}\left\{V^{2} \sin (L t)+L \cos (L t)\right\}}{V^{4}+L^{2}} \Gamma(R, V)\right. \\
& \cdot V\} d V \\
& \theta=\frac{\mathrm{Gc}(K-S)}{L^{2}}\left\{1+\frac{2}{\pi} \int_{0}^{\infty} e^{-V^{2} t} \Gamma(R, V) \frac{d V}{V}\right\} \\
& +\frac{S(\mathrm{Gr}+\mathrm{Gc})}{2 L^{2}}\left\{\frac{K_{0}(R \sqrt{-i L})}{K_{0}(\sqrt{-i L})}+\frac{K_{0}(R \sqrt{i L})}{K_{0}(\sqrt{i L})}\right\} \\
& +\frac{2 \mathrm{~S}(\mathrm{Gr}+\mathrm{Gc})}{\pi L^{2}} \\
& \cdot \int_{0}^{\infty} \frac{e^{-V^{2} t}\left\{V^{2} \cos (L t)-L \sin (L t)\right\}}{V^{4}+L^{2}} \Gamma(R, V) V d V, \\
& \phi=\frac{\mathrm{Gr}(S-K)}{L^{2}}\left\{1+\frac{2}{\pi} \int_{0}^{\infty} e^{-V^{2} t} \Gamma(R, V) \frac{d V}{V}\right\} \\
& +\frac{K(\mathrm{Gr}+\mathrm{Gc})}{2 L^{2}}\left\{\frac{K_{0}(R \sqrt{-i L})}{K_{0}(\sqrt{-i L})}+\frac{K_{0}(R \sqrt{i L})}{K_{0}(\sqrt{i L})}\right\} \\
& +\frac{2 K(\mathrm{Gr}+\mathrm{Gc})}{\pi L^{2}} \\
& \cdot \int_{0}^{\infty}\left\{\frac{e^{-V^{2} t}\left\{V^{2} \cos (L t)-L \sin (L t)\right\}}{V^{4}+L^{2}} \Gamma(R, V)\right. \\
& \cdot V\} d V
\end{aligned}
$$

where $L^{2}=S \mathrm{Gr}+K \mathrm{Gc}$. It is to be noted that $i=$ $\sqrt{-1}$ and in expressions $U, \theta$, and $\phi$ above, the expressions with complex quantities appear along with their conjugates, thereby resulting in real quantities. This kind of appearance is also present in our forgoing analysis. MATHEMATICA is used to deal with these situations during computations.

3.1. Skin Friction. The nondimensional skin friction $\tau=$ $-\left.(\partial U / \partial R)\right|_{R=1}$ is obtained from the velocity profile (10) as

$$
\begin{aligned}
\tau= & \frac{\mathrm{Gr}+\mathrm{Gc}}{2 L i}\left\{\frac{\sqrt{-i L} K_{1}(\sqrt{-i L})}{K_{0}(\sqrt{-i L})}-\frac{\sqrt{i L} K_{1}(\sqrt{i L})}{K_{0}(\sqrt{i L})}\right\} \\
& +\frac{2(\mathrm{Gr}+\mathrm{Gc})}{\pi L} \\
& \cdot \int_{0}^{\infty} \frac{e^{-V^{2} t}\left\{V^{2} \sin (L t)+L \cos (L t)\right\}}{V^{4}+L^{2}} \Gamma_{1}(V) V^{2} d V .
\end{aligned}
$$


3.2. Nusselt Number. The nondimensional Nusselt number $\mathrm{Nu}=-\left.(\partial \theta / \partial R)\right|_{R=1}$ is obtained from the temperature profile (11) as

$$
\begin{aligned}
\mathrm{Nu} & =\frac{2 \mathrm{Gc}(K-S)}{\pi L^{2}} \int_{0}^{\infty} e^{-V^{2} t} \Gamma_{1}(V) d V \\
& +\frac{S(\mathrm{Gr}+\mathrm{Gc})}{2 L^{2}}\left\{\frac{\sqrt{i L} K_{1}(\sqrt{i L})}{K_{0}(\sqrt{i L})}\right. \\
& \left.+\frac{\sqrt{-i L} K_{1}(\sqrt{-i L})}{K_{0}(\sqrt{-i L})}\right\}+\frac{2 S(\mathrm{Gr}+\mathrm{Gc})}{\pi L^{2}} \\
& \cdot \int_{0}^{\infty} \frac{e^{-V^{2} t}\left\{V^{2} \cos (L t)-L \sin (L t)\right\}}{V^{4}+L^{2}} \Gamma_{1}(V) V^{2} d V
\end{aligned}
$$

3.3. Sherwood Number. The nondimensional Sherwood number $\mathrm{Sh}=-\left.(\partial \phi / \partial R)\right|_{R=1}$ is obtained from the concentration profile (12) as

$$
\begin{aligned}
\mathrm{Sh} & =\frac{2 \mathrm{Gr}(S-K)}{\pi L^{2}} \int_{0}^{\infty} e^{-V^{2} t} \Gamma_{1}(V) d V \\
& +\frac{S(\mathrm{Gr}+\mathrm{Gc})}{2 L^{2}}\left\{\frac{\sqrt{i L} K_{1}(\sqrt{i L})}{K_{0}(\sqrt{i L})}\right. \\
& \left.+\frac{\sqrt{-i L} K_{1}(\sqrt{-i L})}{K_{0}(\sqrt{-i L})}\right\}+\frac{2 S(\mathrm{Gr}+\mathrm{Gc})}{\pi L^{2}} \\
& \cdot \int_{0}^{\infty} \frac{e^{-V^{2} t}\left\{V^{2} \cos (L t)-L \sin (L t)\right\}}{V^{4}+L^{2}} \Gamma_{1}(V) V^{2} d V
\end{aligned}
$$

where

$$
\begin{gathered}
\Gamma(R, V)=\frac{J_{0}(R V) Y_{0}(V)-Y_{0}(R V) J_{0}(V)}{J_{0}^{2}(V)+Y_{0}^{2}(V)}, \\
\Gamma_{1}(V)=\frac{J_{1}(V) Y_{0}(V)-Y_{1}(V) J_{0}(V)}{J_{0}^{2}(V)+Y_{0}^{2}(V)} .
\end{gathered}
$$

\section{Steady State Solution}

Steady state equations are obtained by neglecting the time derivative terms from (4). Solving these equations and using boundary conditions (5) we obtain the expressions of velocity, temperature, and concentration profiles as

$$
U_{S}=\frac{\mathrm{Gr}+\mathrm{Gc}}{2 L i}\left\{\frac{K_{0}(R \sqrt{-i L})}{K_{0}(\sqrt{-i L})}-\frac{K_{0}(R \sqrt{i L})}{K_{0}(\sqrt{i L})}\right\}
$$

$$
\begin{aligned}
\theta_{S} & \frac{\mathrm{Gc}(K-S)}{L^{2}} \\
& +\frac{S(\mathrm{Gr}+\mathrm{Gc})}{2 L^{2}}\left\{\frac{K_{0}(R \sqrt{-i L})}{K_{0}(\sqrt{-i L})}+\frac{K_{0}(R \sqrt{i L})}{K_{0}(\sqrt{i L})}\right\}, \\
\phi_{S} & \quad \frac{\mathrm{Gr}(S-K)}{L^{2}} \\
& +\frac{K(\mathrm{Gr}+\mathrm{Gc})}{2 L^{2}}\left\{\frac{K_{0}(R \sqrt{-i L})}{K_{0}(\sqrt{-i L})}+\frac{K_{0}(R \sqrt{i L})}{K_{0}(\sqrt{i L})}\right\} .
\end{aligned}
$$

4.1. Skin Friction. Nondimensional skin friction $\tau_{S}=$ $-\left.\left(\partial U_{S} / \partial R\right)\right|_{R=1}$ is obtained from velocity profile (17) as

$\tau_{S}$

$$
=\frac{\mathrm{Gr}+\mathrm{Gc}}{2 L i}\left\{\frac{\sqrt{-i L} K_{1}(\sqrt{-i L})}{K_{0}(\sqrt{-i L})}-\frac{\sqrt{i L} K_{1}(R \sqrt{i L})}{K_{0}(\sqrt{i L})}\right\} .
$$

4.2. Nusselt Number. Nondimensional Nusselt number $\mathrm{Nu}_{S}=-\left.\left(\partial \theta_{S} / \partial R\right)\right|_{R=1}$ is obtained from temperature profile (18) as

$$
\begin{aligned}
\mathrm{Nu}_{S} & =\frac{S(\mathrm{Gr}+\mathrm{Gc})}{2 L^{2}}\left\{\frac{\sqrt{i L} K_{1}(\sqrt{i L})}{K_{0}(\sqrt{i L})}\right. \\
& \left.+\frac{\sqrt{-i L} K_{1}(\sqrt{-i L})}{K_{0}(\sqrt{-i L})}\right\} .
\end{aligned}
$$

4.3. Sherwood Number. Nondimensional Sherwood number $\mathrm{Sh}_{S}=-\left.\left(\partial \phi_{S} / \partial R\right)\right|_{R=1}$ is obtained from concentration profile (19) as

$$
\begin{aligned}
\mathrm{Sh}_{S} & =\frac{K(\mathrm{Gr}+\mathrm{Gc})}{2 L^{2}}\left\{\frac{\sqrt{i L} K_{1}(\sqrt{i L})}{K_{0}(\sqrt{i L})}\right. \\
& \left.+\frac{\sqrt{-i L} K_{1}(\sqrt{-i L})}{K_{0}(\sqrt{-i L})}\right\} .
\end{aligned}
$$

It is to be noted that as $t \rightarrow \infty$ the expressions for unsteady velocity, temperature, concentration, skin friction, Nusselt number, and Sherwood number given by (10), (11), (12), (13), (14), and (15), respectively, approach to the corresponding expressions for steady state given by (17), (18), (19), (20), (21), and (22).

\section{Boundary Layer Thickness}

The peak vertical velocity in the steady state occurs at a nondimensional distance $R_{\delta}$, from the surface of the cylinder 
TABLE 1: Boundary layer thickness for various $S, K, \mathrm{Gr}$, and Gc.

\begin{tabular}{lcccc}
\hline Gr & Gc & $S$ & $K$ & $R_{\delta}$ \\
\hline 5 & 5 & 0.2 & 0 & 1.890311 \\
5 & 5 & 0 & 0.4 & 1.764592 \\
5 & 5 & 0.2 & 0.4 & 1.699095 \\
5 & 5 & 0.2 & 0.6 & 1.655900 \\
5 & 5 & 0.6 & 0.4 & 1.624154 \\
8 & 5 & 0.2 & 0.4 & 1.671416 \\
10 & 5 & 0.2 & 0.4 & 1.655901 \\
5 & 8 & 0.2 & 0.4 & 1.648831 \\
5 & 10 & 0.2 & 0.4 & 1.624154 \\
\hline
\end{tabular}

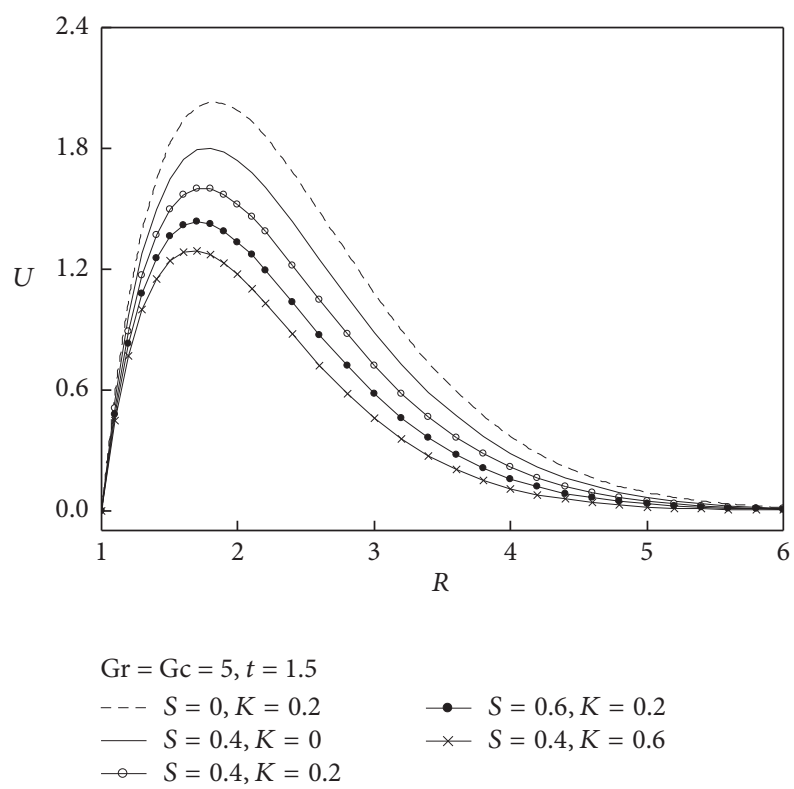

FIGURE 2: Effects of thermal and mass stratification on velocity profile at $\mathrm{Gr}=5$ and $\mathrm{Gc}=5$.

where $R_{\delta}$ can be obtained from $\left.\left(d U_{S} / d R\right)\right|_{R=R_{\delta}}=0$. This leads to the determination of $R_{\delta}$ from the following expression:

$$
\begin{aligned}
& (1+i) K_{0}(\sqrt{i L}) K_{1}\left(R_{\delta} \sqrt{-i L}\right) \\
& \quad+(1-i) K_{0}(\sqrt{-i L}) K_{1}\left(R_{\delta} \sqrt{+i L}\right)=0 .
\end{aligned}
$$

A list of calculated values of $R_{\delta}$ for different values $S, K$, Gr, and Gc is tabulated in Table 1.

\section{Results and Discussion}

In order to understand the physical insight of the problem, numerical computations for velocity, temperature, concentration, skin friction, Nusselt number, and Sherwood number are made for various physical parameters and presented in figures. Solutions of unsteady state for larger time are compared with the solutions of steady state.

Velocity profiles represented by Figure 2 show the effects of $S$ and $K$ for $\mathrm{Gr}=\mathrm{Gc}=5$ and $t=1.5$ and Figure 3 shows the effects of $\mathrm{Gr}$ and $\mathrm{Gc}$ for $S=0.4, K=0.2$, and $t=1.5$. It is

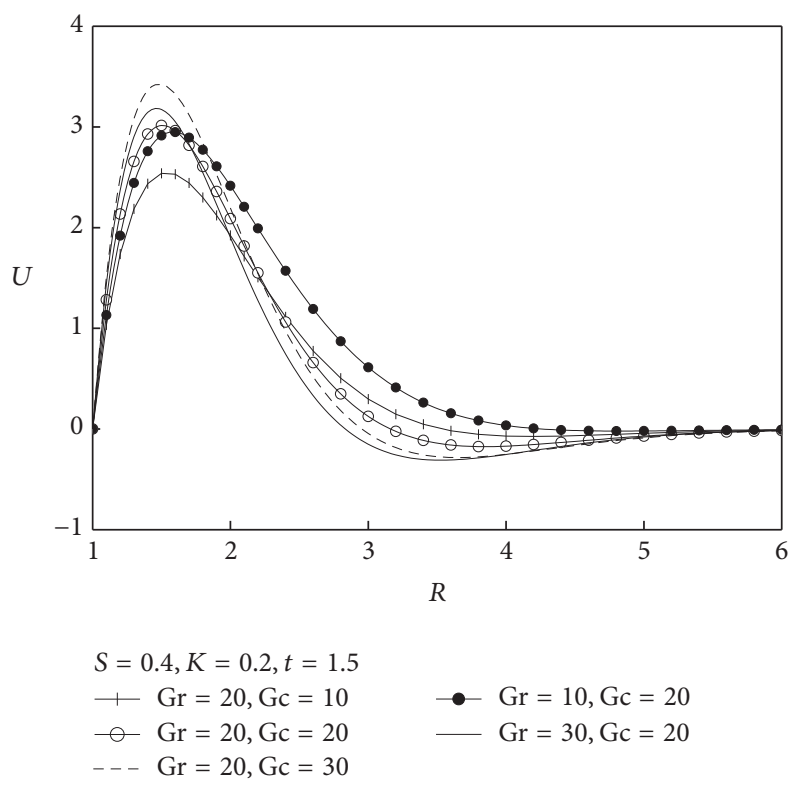

FIGURE 3: Effects of Gr and Gc on velocity profile at $S=0.4$ and $K=0.2$.

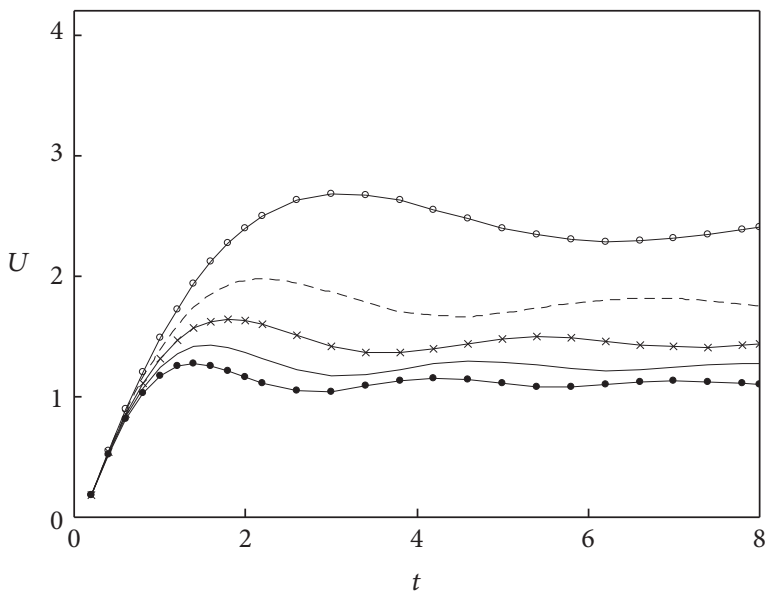

$$
\begin{aligned}
& \mathrm{Gr}=\mathrm{Gc}=5, R=1.8 \\
& \because S=0, K=0.2 \quad-S=0.6, K=0.2 \\
& --S=0.4, K=0 \quad \longrightarrow S=0.4, K=0.6 \\
& \star \quad S=0.4, K=0.2
\end{aligned}
$$

FIGURE 4: Effects of $S$ and $K$ on velocity profile against time at $\mathrm{Gr}=$ 5 and $\mathrm{Gc}=5$.

observed from these figures that velocity decreases with the increase in $S$ or increase in $K$ but increases with increase in Gr or Gc. As mentioned by Shapiro and Fedorovich [15], the fluid leads to a stably stratified flow by decreasing the fluid velocity with thermal stratification $S>0$. Remarkably, in our analysis, we have seen that, for mass stratification $K>0$, the fluid velocity still reduces. Therefore, we conclude that the inclusion of mass stratification also leads to a stably stratified flow. Figures 4 and 5, respectively, show the effects of $S, K$ and $\mathrm{Gr}, \mathrm{Gc}$ on velocity profiles against time at $R=1.8$. The time required to reach the steady state increases with decrease in 


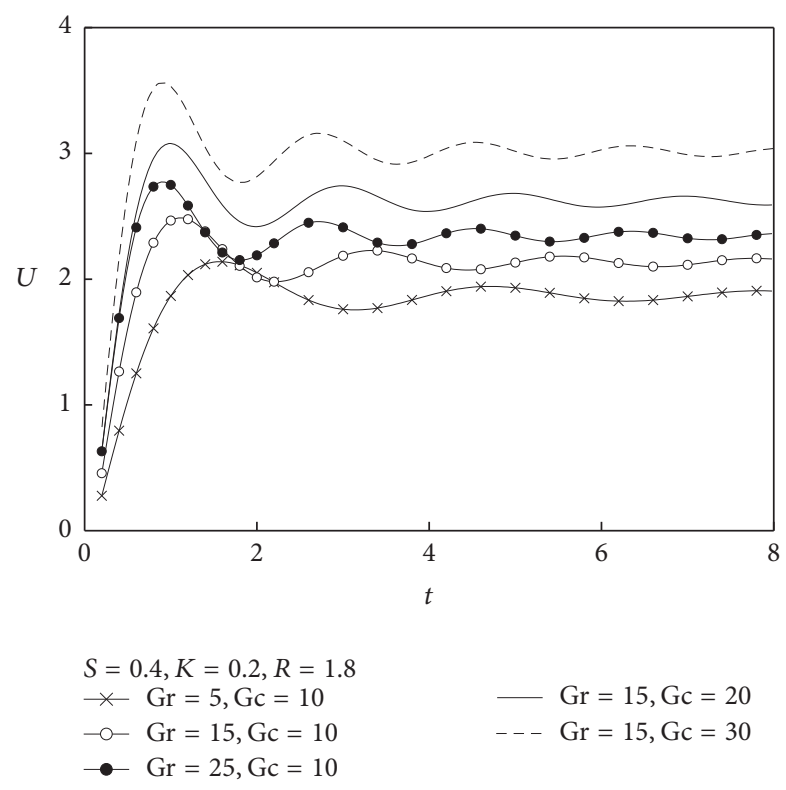

FIgURE 5: Effects of $\mathrm{Gr}$ and Gc on velocity profile against time at $S=0.4$ and $K=0.2$.

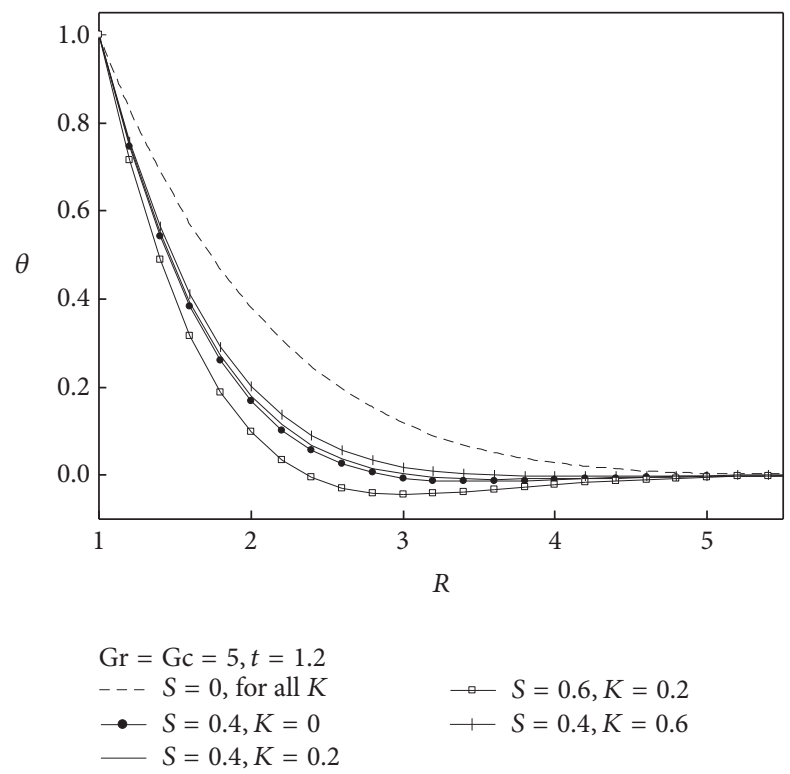

FIGURE 6: Effects of $S$ and $K$ on temperature profile at $\mathrm{Gr}=5$ and Gc $=5$.

stratification parameters $S$ or $K$ and increases with increase in the values of Gr or Gc.

Figure 6 shows the effects of thermal and mass stratification on temperature profiles at $\mathrm{Gr}=\mathrm{Gc}=5$ at $t=1.2$. It is observed from the figure that temperature decreases with the increase in the stratification parameter $S$ or $K$. Also, temperature becomes negative in presence of dense stratified fluid. This type of behaviour has also been observed by earlier investigators (Kulkarni et al. [26], Loganathan and Ganesan [14], and Deka and Paul [24]). This is because the fluid with dense stratification near the cylinder can

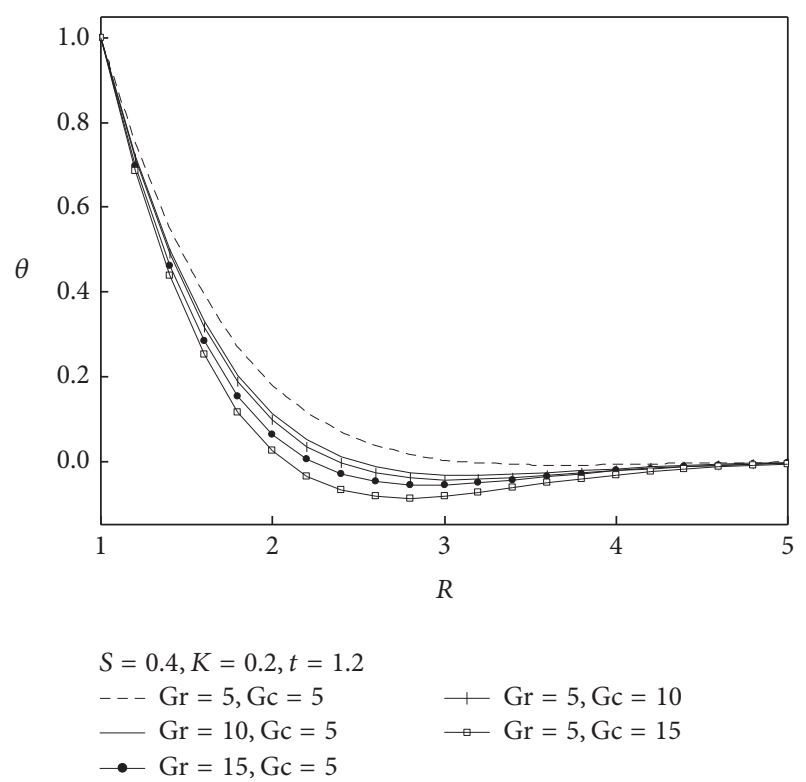

FIGURE 7: Effects of Gr and Gc on temperature profile at $S=0.4$ and $K=0.2$.

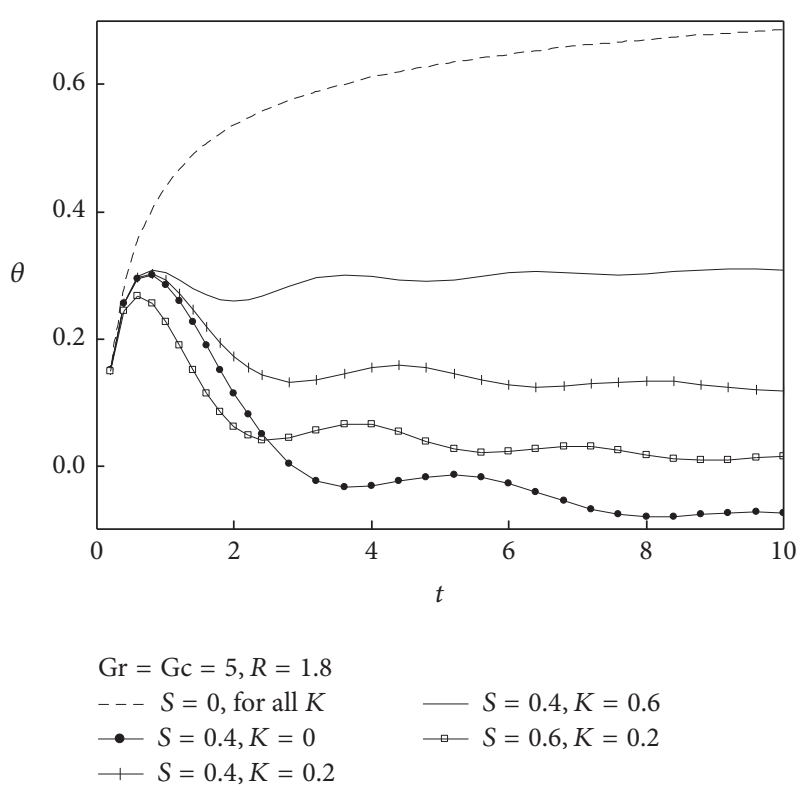

FIGURE 8: Effects of $S$ and $K$ on temperature profile against time at $\mathrm{Gr}=\mathrm{Gc}=5$ for $R=1.8$.

have temperature and concentration lower than the ambient. Figure 7 represents the effects of thermal Grashof number and mass Grashof number on temperature profiles at $S=0.4$, $K=0.2$ for $t=1.2$ and it shows that temperature decreases with increase in $\mathrm{Gr}$ or Gc. Figures 8 and 9, respectively, show the effects of $S, K$ and $\mathrm{Gr}, \mathrm{Gc}$ on temperature profile against time at $R=1.8$. From these figures, it is observed that initially temperature increases sharply with time but for larger time it becomes steady. Also, time required to reach the steady state increases with decrease in $S, K, \mathrm{Gr}$, or Gc. It is also observed 


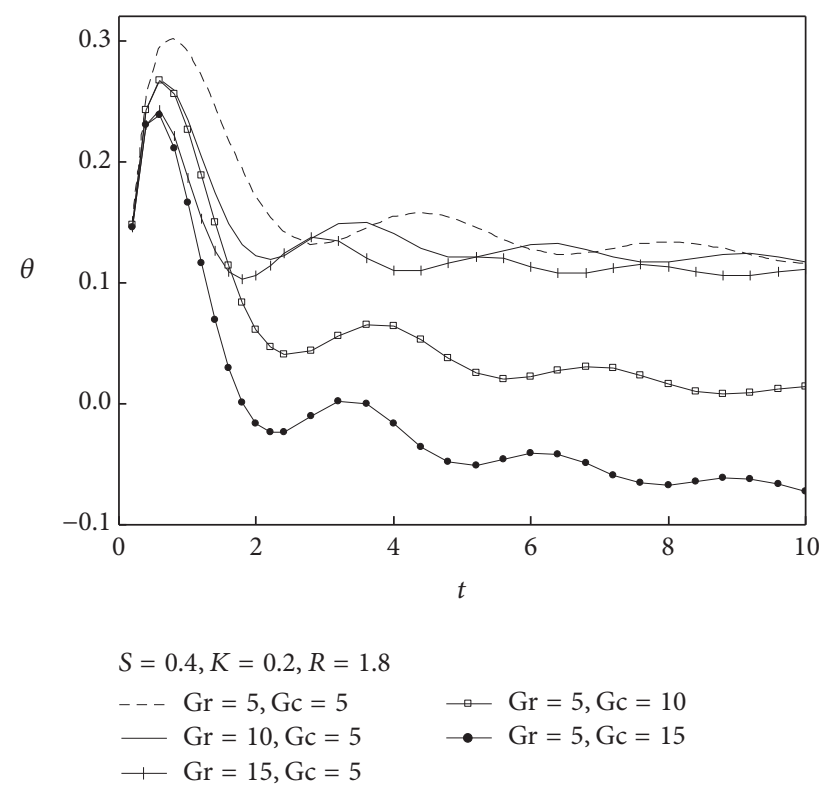

FIGURE 9: Effects of $\mathrm{Gr}$ and $\mathrm{Gc}$ on temperature profile against time at $\mathrm{Gr}=5, \mathrm{Gc}=5$.

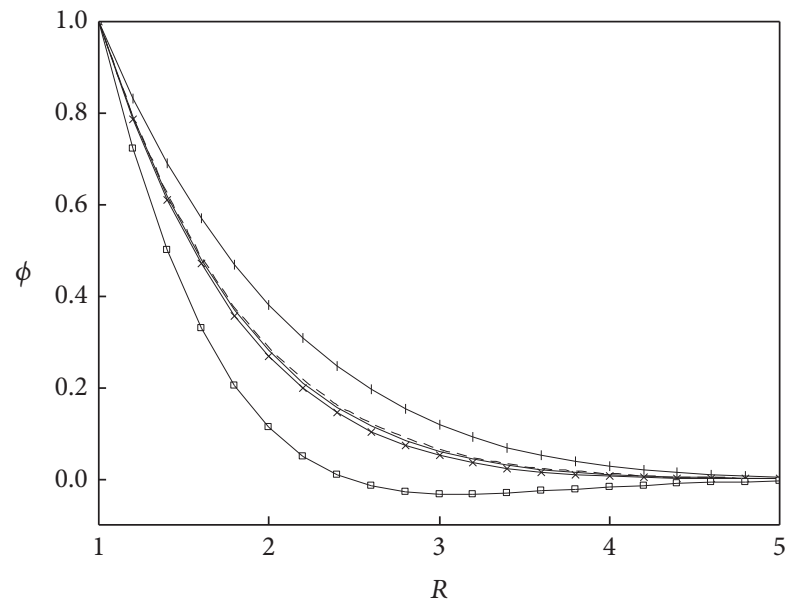

$$
\begin{aligned}
& \mathrm{Gr}=\mathrm{Gc}=5, t=1.5 \\
& +K=0, \text { for all } S \\
& \times K=0.2, S=0
\end{aligned}
$$$$
-K=0.2, S=0.4
$$

FIGURE 10: Effects of $S$ and $K$ on concentration profile at $\mathrm{Gr}=5$, Gc $=5$.

that, in absence of thermal stratification parameter, effect of mass stratification on temperature profile is negligible.

Effects of thermal and mass stratification on concentration profiles are shown in Figure 10 at $\mathrm{Gr}=\mathrm{Gc}=5$ for $t=1.5$. It is observed from the figure that concentration decreases with an increase in stratification parameter $S$ or $K$. Also concentration becomes negative in presence of dense stratified fluid. Figure 11 shows the effects of thermal Grashof number and mass Grashof number on concentration profiles at $S=0.4, K=0.2$ for $t=1.5$ and that concentration decreases with increase in Gr or Gc. Figures 12 and 13,

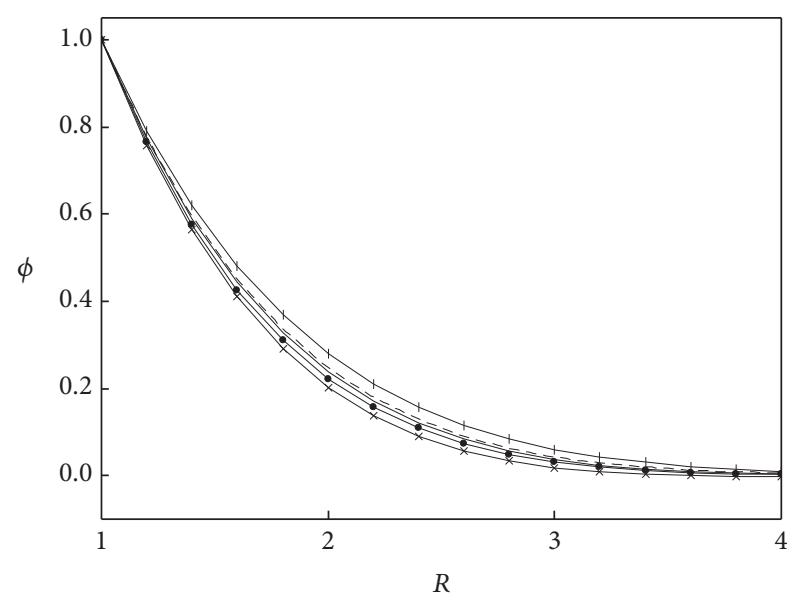

$$
\begin{array}{ll}
S=0.4, K=0.2, t=1.5 & \\
-\quad \mathrm{Gr}=5, \mathrm{Gc}=5 & -\mathrm{Gr}=5, \mathrm{Gc}=10 \\
--\mathrm{Gr}=10, \mathrm{Gc}=5 & \ngtr \mathrm{Gr}=5, \mathrm{Gc}=15 \\
-\mathrm{Gr}=15, \mathrm{Gc}=5 &
\end{array}
$$

FIGURE 11: Effects of $\mathrm{Gr}$ and $\mathrm{Gc}$ on concentration profile at $S=0.4$, $K=0.2$.

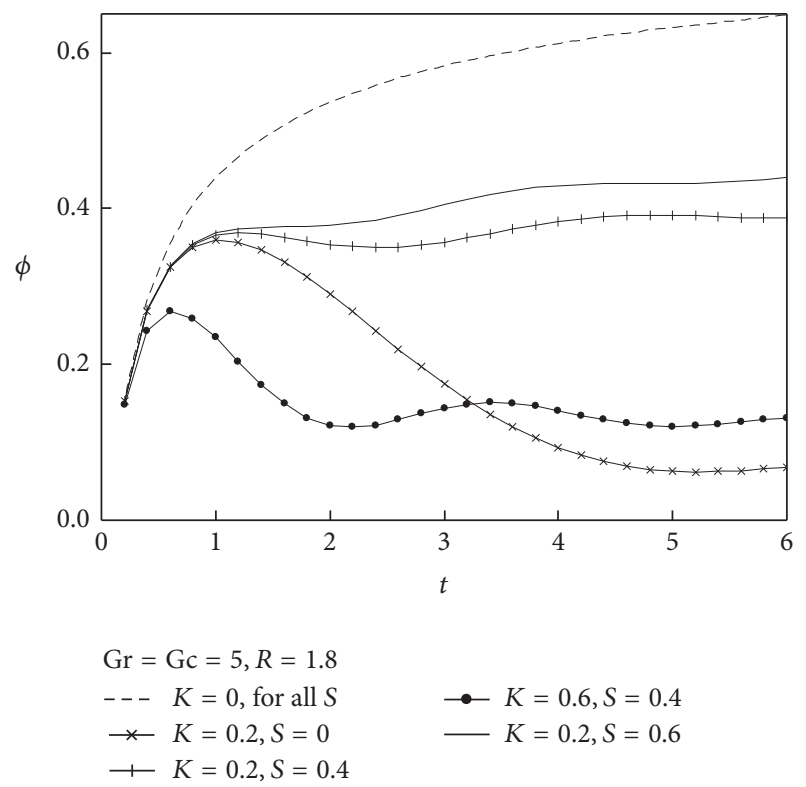

FIGURE 12: Effects of $S$ and $K$ on concentration profile against time at $\mathrm{Gr}=5, \mathrm{Gc}=5$.

respectively, show the effects of $S, K$ and Gr, Gc on concentration profiles against time at $R=1.8$. It is found from these figures that initially concentration increases sharply with time but for larger time it becomes steady. Also, time required to reach the steady state increases with decrease in $S, K, \mathrm{Gr}$, or Gc. It is also observed that, in absence of mass stratification parameter, effect of thermal stratification on concentration profile is negligible.

Skin friction represented by Figures 14 and 15 shows the effects of $S, K$ and $\mathrm{Gr}, \mathrm{Gc}$, respectively. It is observed from these figures that skin friction initially decreases with time 


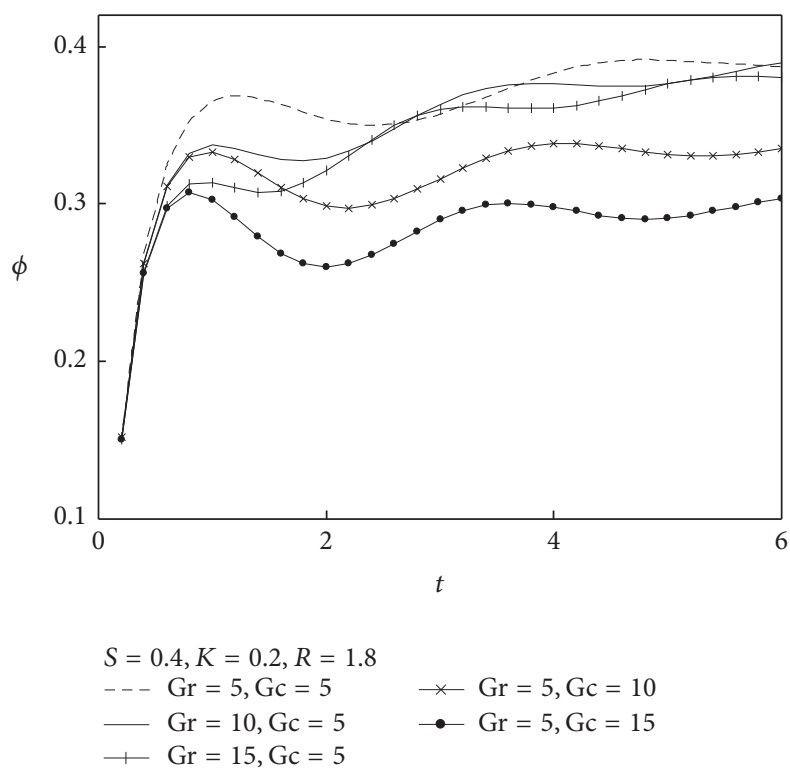

FIGURE 13: Effects of Gr and Gc on concentration profile against time at $S=0.4, K=0.2$.

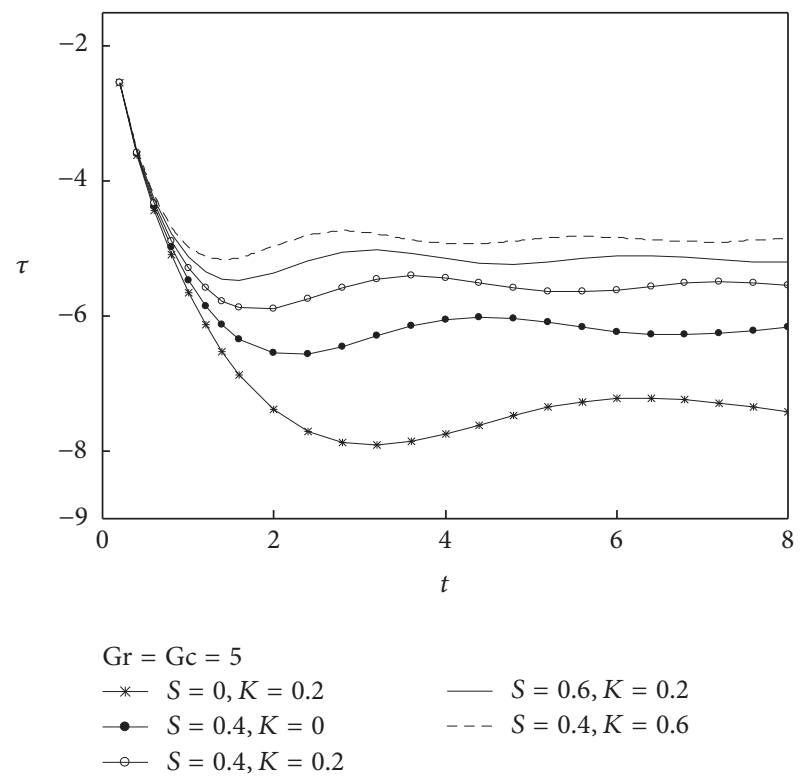

FIGURE 14: Effects of thermal and mass stratification on skin friction at $\mathrm{Gr}=5, \mathrm{Gc}=5$.

but becomes steady at large time. Skin friction increases with an increase in thermal or mass stratification but decreases with an increase in Gr or Gc.

Figures 16 and 17, respectively, depict the effects of thermal stratification and mass stratification and thermal Grashof number and mass Grashof number on Nusselt number against time. It is found that initially Nusselt number decreases very sharply but after certain time it becomes steady. Nusselt number increases with an increase in thermal or mass stratification but decreases with an increase in thermal Grashof number or mass Grashof number. Also

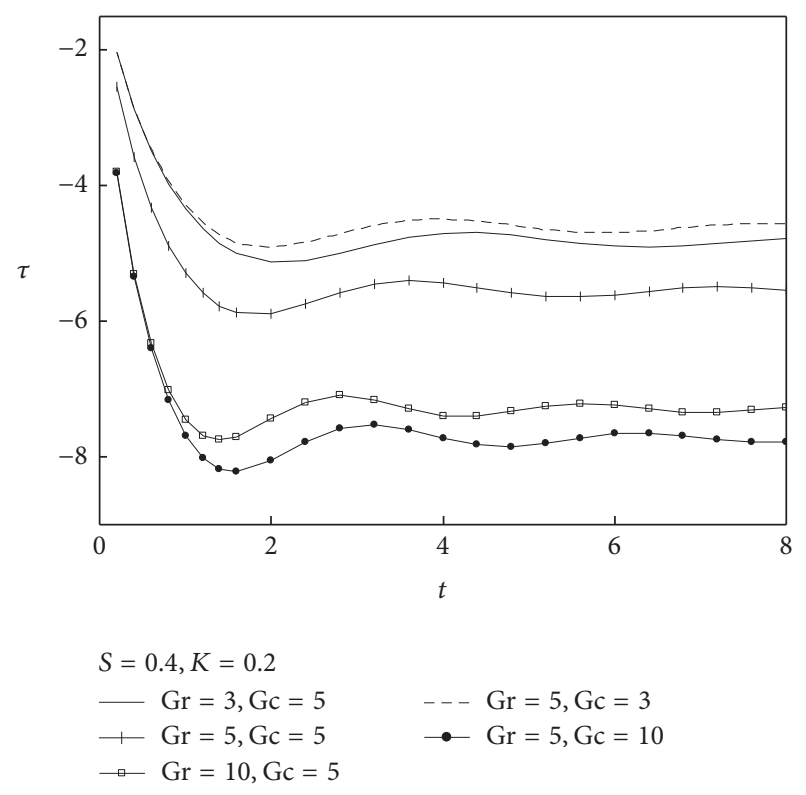

Figure 15: Effects of $\mathrm{Gr}$ and Gc on skin friction for $S=0.4$ and $K=0.2$.

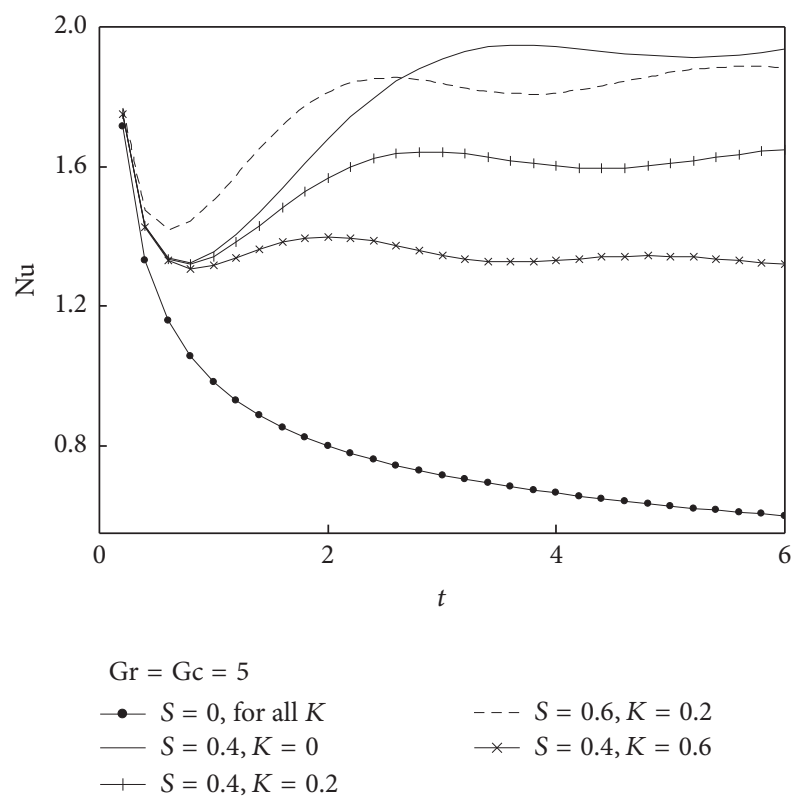

Figure 16: Effects of $S$ and $K$ on Nusselt number for $\mathrm{Gr}=5$ and Gc $=5$.

effect of mass stratification on Nusselt number is negligible, when thermal stratification is absent.

Figures 18 and 19, respectively, show the effect of thermal stratification and mass stratification and thermal Grashof number and mass Grashof number on Sherwood number, that is, rate of mass transfer. It is observed that initially Sherwood number decreases sharply but becomes steady after certain time. Sherwood number increases with an increase in thermal stratification or mass stratification but decreases with an increase in thermal Grashof number or 


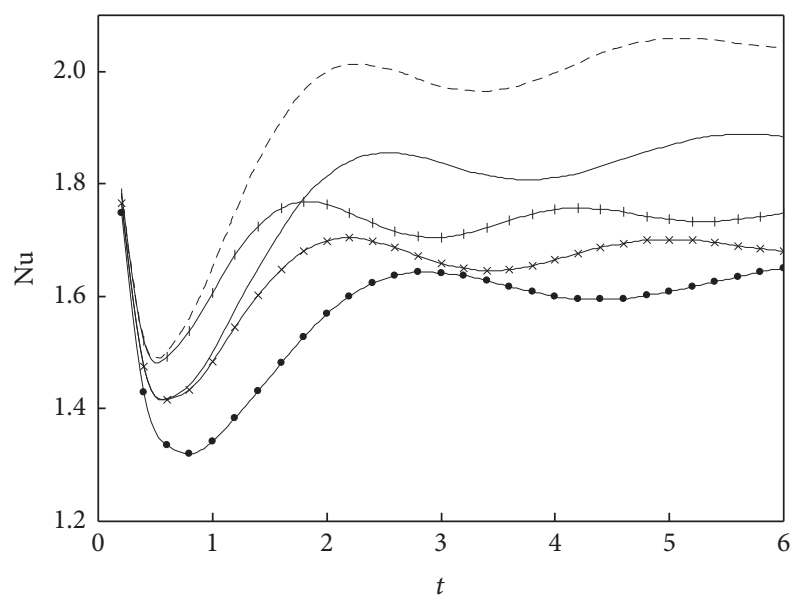

$$
\begin{array}{ll}
S=0.4, K=0.2 & \\
-\mathrm{Gr}=5, \mathrm{Gc}=5 & -\mathrm{Gr}=5, \mathrm{Gc}=10 \\
-\mathrm{Gr}=10, \mathrm{Gc}=5 & --\mathrm{Gr}=5, \mathrm{Gc}=15 \\
\longrightarrow \mathrm{Gr}=15, \mathrm{Gc}=5 &
\end{array}
$$

FIgURE 17: Effects of Gr and Gc on Nusselt number for $S=0.4$ and $K=0.2$.

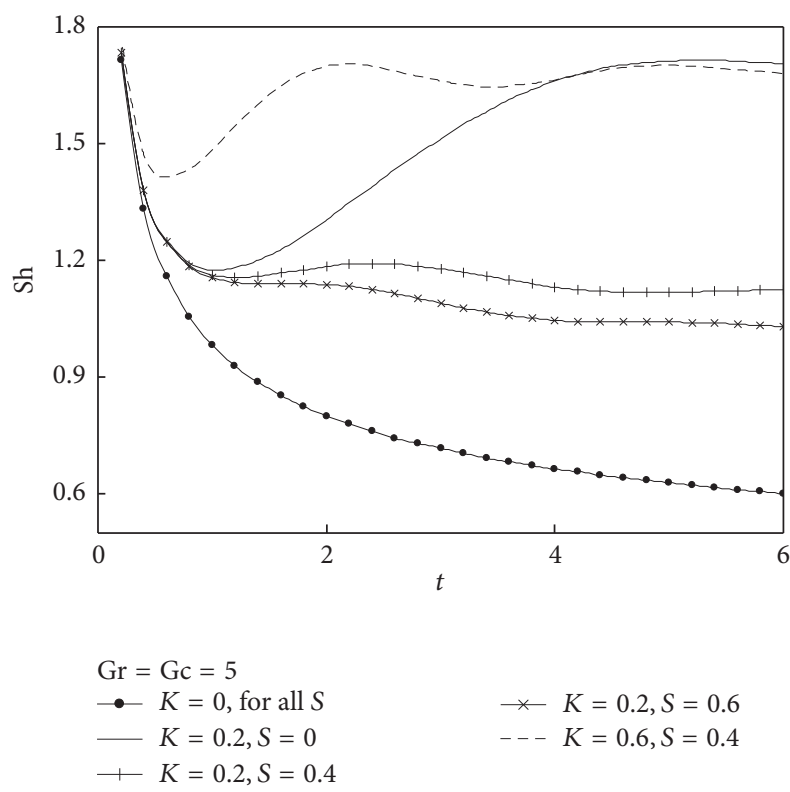

Figure 18: Effects of $S$ and $K$ on Sherwood number for $\mathrm{Gr}=5$ and $\mathrm{Gc}=5$.

mass Grashof number. Also, effect of thermal stratification on rate of mass transfer is negligible when the fluid is free from mass stratification. A decrease in the thermal stratification parameter or the concentration stratification parameter leads to a decrease in the Nusselt number and Sherwood number and this trend becomes more pronounced as the fluid moves downstream (Figure 2). This is due to the fact that increasing the thermal and concentration stratification parameters decreases the buoyancy force due to thermal and solutal

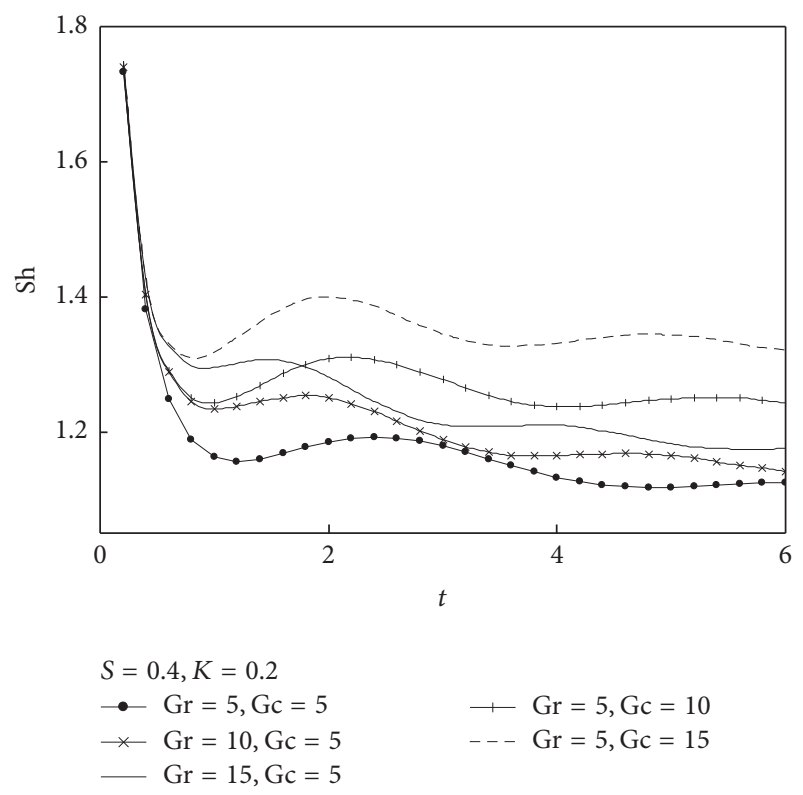

FIGURE 19: Effects of Gr and Gc on Sherwood number for $S=0.4$ and $K=0.2$.

gradients and retards the flow, increasing the thermal and concentration boundary layer thickness and thus decreasing the heat and mass transfer rates between the fluid and the cylinder wall.

\section{Conclusions}

(i) Solution expressions obtained for unsteady state approaches to the solutions of steady state as $t$ becomes large.

(ii) The time required to reach steady state velocity increases with increase in Gr or Gc but decreases with increase in $S$ or $K$, while time required to reach the steady state temperature and concentration increases with decrease in $S, K, \mathrm{Gr}$, or Gc.

(iii) Velocity increases with increase in Gr or Gc but decreases with increase in $S$ or $K$.

(iv) Temperature decreases with increase in $S, K, \mathrm{Gr}$, or Gc. In presence of high stratification temperature becomes negative.

(v) Concentration decreases with increase in $S, K$, Gr, or Gc. In presence of high stratification concentration becomes negative.

(vi) Skin friction decreases with increase in Gr or Gc but increases with increase in $S$ or $K$.

(vii) Rate of heat transfer increases as $\mathrm{Gr}, K, S$, or $K$ increases.

(viii) Rate of mass transfer increases with increase in $\mathrm{Gr}, K$, $S$, or $K$. 


\section{Appendix}

Laplace transform of (4) with initial conditions in (5) and with $\operatorname{Pr}=\mathrm{Sc}=1$ is (6), (7), and (8) with transformed boundary conditions

$$
\begin{aligned}
& \bar{U}=0, \\
& \bar{\theta}=\frac{1}{p} \\
& \bar{\phi}=\frac{1}{p}
\end{aligned}
$$$$
\text { at } R=1 \text {, }
$$

$$
\begin{aligned}
& \bar{U} \longrightarrow 0 \\
& \bar{\theta} \longrightarrow 0 \\
& \bar{\phi} \longrightarrow 0
\end{aligned}
$$

as $R \longrightarrow \infty$,

where $\bar{U}(p, R), \bar{T}(p, R)$, and $\bar{C}(p, R)$ are Laplace transforms of $U(t, R), T(t, R)$, and $C(t, R)$, respectively, and $p$ is Laplace transform parameter.

Upon applying (7) and (8), we eliminate $\bar{\theta}$ and $\bar{\phi}$ from (6) that give rise to

$$
\left\{\frac{d^{2}}{d R^{2}}+\frac{1}{R} \frac{d}{d R}-a^{2}\right\}\left\{\frac{d^{2}}{d R^{2}}+\frac{1}{R} \frac{d}{d R}-b^{2}\right\} \bar{U}=0
$$

where $a^{2}=p+i L, b^{2}=p-i L, L^{2}=S \mathrm{Gr}+K \mathrm{Sc}$.

Now to determine $\bar{U}$ from (A.2), we rewrite (A.2) as

$$
\left\{\frac{d^{2}}{d R^{2}}+\frac{1}{R} \frac{d}{d R}-a^{2}\right\} W=0
$$

with

$$
\left\{\frac{d^{2}}{d R^{2}}+\frac{1}{R} \frac{d}{d R}-b^{2}\right\} \bar{U}=W
$$

Equation (A.3) is the modified Bessel equation of order zero and its solution is

$$
W=C_{1} I_{0}(a R)+C_{2} K_{0}(a R)
$$

where $C_{1}$ and $C_{2}$ are arbitrary constants. In view of (A.5) and using the following identities (see Carslaw and Jaeger [25])

$$
\begin{aligned}
& \int R I_{0}(a R) K_{0}(b R) d R \\
& =\frac{R}{a^{2}-b^{2}}\left[a I_{1}(a R) K_{0}(b R)+b I_{0}(a R) K_{1}(b R)\right],
\end{aligned}
$$

$$
\begin{aligned}
& \int R K_{0}(a R) K_{0}(b R) d R \\
& =\frac{R}{a^{2}-b^{2}}\left[b K_{0}(a R) K_{1}(b R)-a K_{0}(b R) K_{1}(a R)\right] \\
& \int R I_{0}(a R) I_{0}(b R) d R \\
& =\frac{R}{a^{2}-b^{2}}\left[a I_{0}(b R) I_{1}(a R)-b I_{0}(a R) I_{1}(b R)\right] \\
& I_{0}(a x) K_{1}(a x)+I_{1}(a x) K_{0}(a x)=\frac{1}{a x}
\end{aligned}
$$

the expression for $\bar{U}$ is obtained after applying variation of parameter technique as

$$
\begin{aligned}
\bar{U}= & \frac{C_{1} I_{0}(a R)}{2 i L}+\frac{C_{2} K_{0}(a R)}{2 i L}+C_{3} I_{0}(b R) \\
& +C_{4} K_{0}(b R)
\end{aligned}
$$

where $C_{3}$ and $C_{4}$ are arbitrary constants.

Now, since $I_{0}(a R)$ and $I_{0}(b R)$ are unbounded as $R \rightarrow \infty$, we set $C_{1}=C_{1}=0$ and so (A.7) is then read as

$$
\bar{U}=\frac{C_{2} K_{0}(a R)}{2 i L}+C_{4} K_{0}(b R) .
$$

Now, using (A.8) we have from (6)

$$
C_{2} K_{0}(a R)-2 i L C_{4} K_{0}(b R)+2 \mathrm{Gr} \bar{\theta}+2 \mathrm{Gc} \bar{\phi}=0 .
$$

Using boundary conditions (A.1) in (A.8) and (A.9) and solving we have

$$
\begin{aligned}
C_{2} & =\frac{-\mathrm{Gr}+\mathrm{Gc}}{p K_{0}(\sqrt{p+i L})} \\
C_{4} & =\frac{\mathrm{Gr}+\mathrm{Gc}}{2 p i L K_{0}(\sqrt{p-i L})}
\end{aligned}
$$

Thus we have

$$
\begin{aligned}
& \bar{U} \\
& =\frac{\mathrm{Gr}+\mathrm{Gc}}{2 i L}\left\{\frac{K_{0}(R \sqrt{p-i L})}{p K_{0}(\sqrt{p-i L})}-\frac{K_{0}(R \sqrt{p+i L})}{p K_{0}(\sqrt{p+i L})}\right\} .
\end{aligned}
$$


Similarly, substituting the expression of $\bar{U}$ in (7) and (8) and solving, the expressions of $\bar{\theta}$ and $\bar{\phi}$ can be obtained as

$$
\begin{aligned}
\bar{\theta}= & \frac{\mathrm{Gc}(K-S)}{L^{2}} \frac{K_{0}(R \sqrt{p})}{p K_{0}(\sqrt{p})} \\
& +\frac{S(\mathrm{Gr}+\mathrm{Gc})}{2 L^{2}}\left\{\frac{K_{0}(R \sqrt{p-i L})}{p K_{0}(\sqrt{p-i L})}\right. \\
& \left.+\frac{K_{0}(R \sqrt{p+i L})}{p K_{0}(\sqrt{p+i L})}\right\}, \\
\bar{\phi} & =\frac{\mathrm{Gr}(S-K)}{L^{2}} \frac{K_{0}}{p K_{0}(\sqrt{p})} \\
& +\frac{K(\mathrm{Gr}+\mathrm{Gc})}{2 L^{2}}\left\{\frac{K_{0}(R \sqrt{p-i L})}{p K_{0}(\sqrt{p-i L})}\right. \\
& \left.+\frac{K_{0}(R \sqrt{p+i L})}{p K_{0}(\sqrt{p+i L})}\right\} .
\end{aligned}
$$

\section{Nomenclature}

$C:$ Concentration

$C_{0}$ : Concentration at the surface of the cylinder

$D:$ Mass diffusion coefficient

Gr: Thermal Grashof number

Gc: Mass Grashof number

g: Acceleration due to gravity

$J_{0}$ : Bessel function of first kind and order zero

$J_{1}$ : Bessel function of first kind and order one

$K$ : Dimensionless mass stratification parameter

$K_{0}$ : Modified Bessel function of second kind and order zero

$K_{1}$ : Modified Bessel function of second kind and order one

$\mathrm{Nu}$ : Nusselt number

Pr: Prandtl number

$r$ : Radial coordinate measured from the axis of the cylinder

$r_{0}$ : Radius of the cylinder

$R:$ Dimensionless radial coordinate

$S$ : Dimensionless thermal stratification parameter

Sc: Schmidt number

Sh: Sherwood number

$t^{\prime}$ : Time

$t$ : Dimensionless time

T: Temperature

$T_{0}$ : Temperature at the surface of the cylinder

$u$ : $z$-component of velocity

$U:$ Dimensionless velocity

$Y_{0}$ : Bessel function of second kind and order zero
$Y_{1}$ : Bessel function of second kind and order one

$\alpha$ : Thermal diffusivity

$\nu$ : Kinematic viscosity

$\beta$ : Volumetric coefficient of thermal expansion

$\beta^{*}$ : Volumetric coefficient of expansion with concentration

$\theta:$ Dimensionless temperature

$\phi$ : Dimensionless concentration

$\gamma:$ Thermal stratification parameter $\left(=d T_{\infty}^{\prime}(z) /\right.$ $\left.d z+g / C_{p}\right)$

$\xi$ : $\quad$ Mass stratification parameter $\left(=d C_{\infty}^{\prime}(z) / d z\right)$.

\section{Competing Interests}

The authors declare that there is no conflict of interests regarding the publication of this paper.

\section{Acknowledgments}

One of the authors acknowledges the support from the University Grants Commission, New Delhi, India.

\section{References}

[1] B. Gebhart and L. Pera, "The nature of vertical natural convection flows resulting from the combined buoyancy effects of thermal and mass diffusion," International Journal of Heat and Mass Transfer, vol. 14, no. 12, pp. 2025-2050, 1971.

[2] F. A. Bottemanne, "Experimental results of pure and simultaneous heat and mass transfer by free convection about a vertical cylinder for Pr=0.71 and Sc=0.63," Applied Scientific Research, vol. 25, no. 5, pp. 372-382, 1972.

[3] T. S. Chen and C. F. Yuh, "Combined heat and mass transfer in natural convection along a vertical cylinder," International Journal of Heat and Mass Transfer, vol. 23, no. 4, pp. 451-461, 1980.

[4] K. Velusamy and V. K. Garg, "Transient natural convection over a heat generating vertical cylinder," International Journal of Heat and Mass Transfer, vol. 35, no. 5, pp. 1293-1306, 1992.

[5] P. Ganesan and H. P. Rani, "Transient natural convection along vertical cylinder with heat and mass transfer," Heat and Mass Transfer, vol. 33, no. 5-6, pp. 449-455, 1998.

[6] P. Ganesan and P. Loganathan, "Effects of mass transfer and flow past a moving vertical cylinder with constant heat flux," Acta Mechanica, vol. 150, no. 3-4, pp. 179-190, 2001.

[7] P. Ganesan and P. Loganathan, "Unsteady natural convective flow past a moving vertical cylinder with heat and mass transfer," Heat and Mass Transfer, vol. 37, no. 1, pp. 59-65, 2001.

[8] P. Ganesan and P. Loganathan, "Heat and mass flux effects on a moving vertical cylinder with chemically reactive species diffusion," Journal of Engineering Physics and Thermophysics, vol. 75, no. 4, pp. 899-909, 2002.

[9] H. P. Rani, "Transient natural convection along a vertical cylinder with variable surface temperature and mass diffusion," Heat and Mass Transfer, vol. 40, no. 1-2, pp. 67-73, 2003.

[10] M. Devakar, D. Sreenivasu, and B. Shankar, "Analytical solutions of couple stress fluid flows with slip boundary conditions," Alexandria Engineering Journal, vol. 53, no. 3, pp. 723-730, 2014. 
[11] R. K. Deka, A. Paul, and A. Chaliha, "Transient free convection flow past vertical cylinder with constant heat flux and mass transfer," Ain Shams Engineering Journal, 2015.

[12] H. S. Takhar, A. J. Chamkha, and G. Nath, "Natural convection flow from a continuously moving vertical surface immersed in a thermally stratified medium," Heat and Mass Transfer/Waermeund Stoffuebertragung, vol. 38, no. 1, pp. 17-24, 2001.

[13] H. S. Takhar, A. J. Chamkha, and G. Nath, "Natural convection on a vertical cylinder embedded in a thermally stratified highporosity medium," International Journal of Thermal Sciences, vol. 41, no. 1, pp. 83-93, 2002.

[14] P. Loganathan and P. Ganesan, "Numerical study of doublediffusive, free convective flow past a moving vertical cylinder," Journal of Engineering Physics and Thermophysics, vol. 79, no. 1, pp. 73-78, 2006.

[15] A. Shapiro and E. Fedorovich, "Unsteady convectively driven flow along a vertical plate immersed in a stably stratified fluid," Journal of Fluid Mechanics, vol. 498, pp. 333-352, 2004.

[16] A. Shapiro and E. Fedorovich, "Prandtl number dependence of unsteady natural convection along a vertical plate in a stably stratified fluid," International Journal of Heat and Mass Transfer, vol. 47, no. 22, pp. 4911-4927, 2004.

[17] C.-Y. Cheng, "Combined heat and mass transfer in natural convection flow from a vertical wavy surface in a powerlaw fluid saturated porous medium with thermal and mass stratification," International Communications in Heat and Mass Transfer, vol. 36, no. 4, pp. 351-356, 2009.

[18] D. Srinivasacharya and G. S. Reddy, "Effect of double stratification on free convection in a power-law fluid saturated porous medium," Fluid Dynamics and Materials Processing, vol. 9, no. 3, pp. 291-305, 2013.

[19] D. Srinivasacharya and C. RamReddy, "Natural convection heat and mass transfer in a micropolar fluid with thermal and mass stratification," International Journal for Computational Methods in Engineering Science and Mechanics, vol. 14, no. 5, pp. 401-413, 2013.

[20] B. V. Rathish Kumar and S. V. S. S. N. V. G. Krishna Murthy, "Double diffusive free convection induced by verticalwavy surface in a doubly stratified Darcy porous medium under the influence of Soret and Dufour effect," Journal of Porous Media, vol. 15, no. 9, pp. 877-890, 2012.

[21] M. Neagu, "Free convective heat and mass transfer induced by a constant heat and mass fluxes vertical wavy wall in a non-Darcy double stratified porous medium," International Journal of Heat and Mass Transfer, vol. 54, no. 11-12, pp. 2310-2318, 2011.

[22] R. K. Deka and A. Paul, "Transient free convection flow past an infinite moving vertical cylinder in a stably stratified fluid," Journal of Heat Transfer, vol. 134, no. 4, Article ID 042503, 8 pages, 2012.

[23] R. K. Deka and A. Paul, "Transient free convection flow past an infinite vertical cylinder with thermal stratification," Journal of Mechanical Science and Technology, vol. 26, no. 8, pp. 2229-2237, 2012.

[24] R. K. Deka and A. Paul, "Convectively driven flow past an infinite moving vertical cylinder with thermal and mass stratification," Pramana-Journal of Physics, vol. 81, no. 4, pp. 641-665, 2013.

[25] H. S. Carslaw and J. C. Jaeger, Operational Methods in Applied Mathematics, Oxford University Press, London, UK, 2nd edition, 1948.

[26] A. K. Kulkarni, H. R. Jacobs, and J. J. Hwang, "Similarity solution for natural convection flow over an isothermal vertical wall immersed in thermally stratified medium," International Journal of Heat and Mass Transfer, vol. 30, no. 4, pp. 691-698, 1987. 


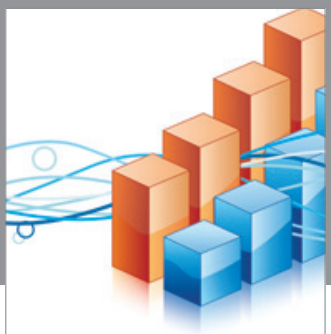

Advances in

Operations Research

vatem alat4

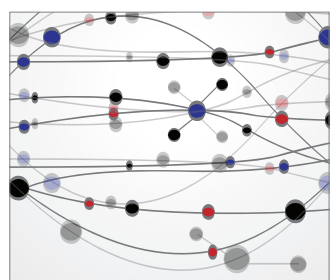

\section{The Scientific} World Journal
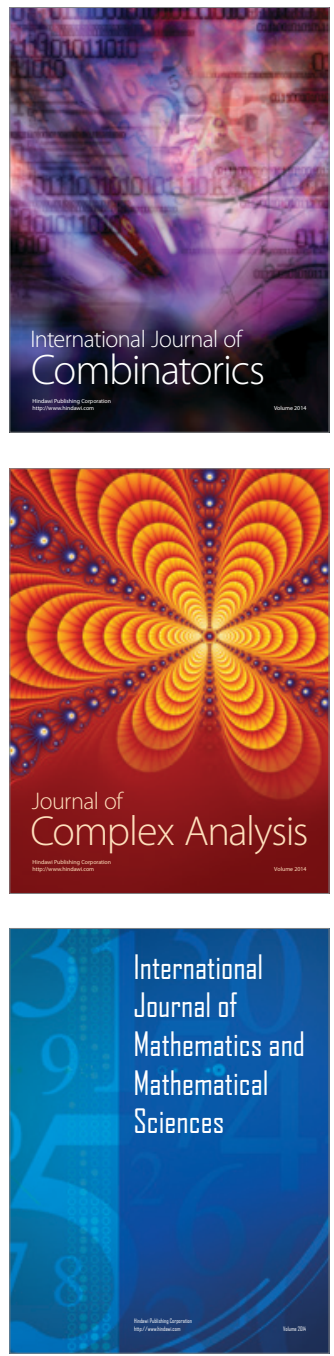
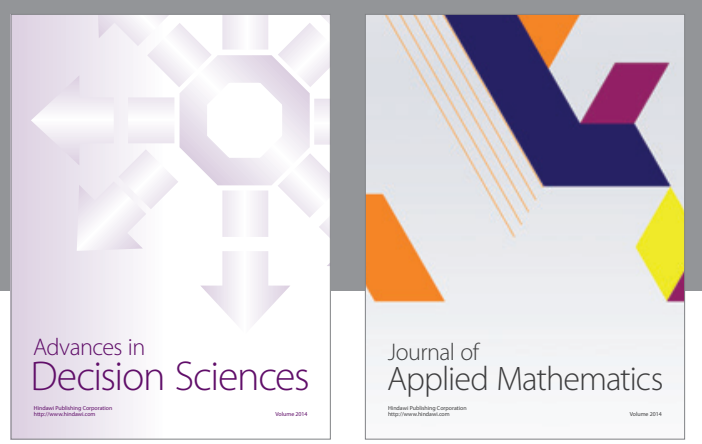

Algebra

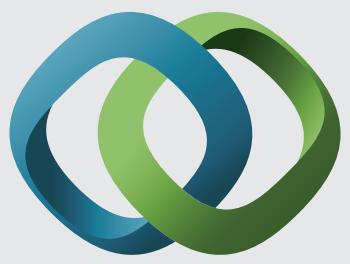

\section{Hindawi}

Submit your manuscripts at

https://www.hindawi.com
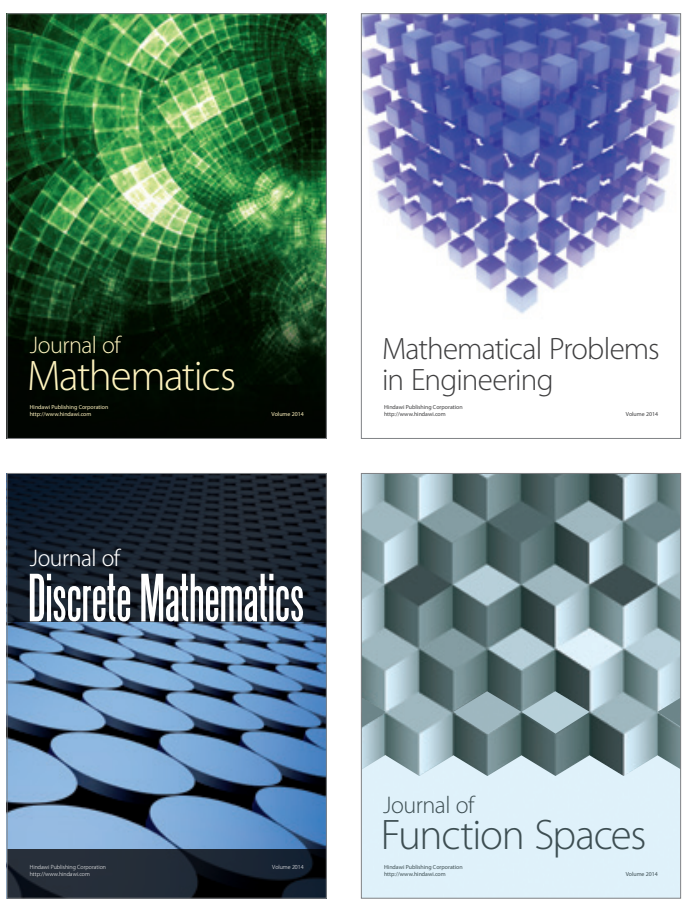

Mathematical Problems in Engineering
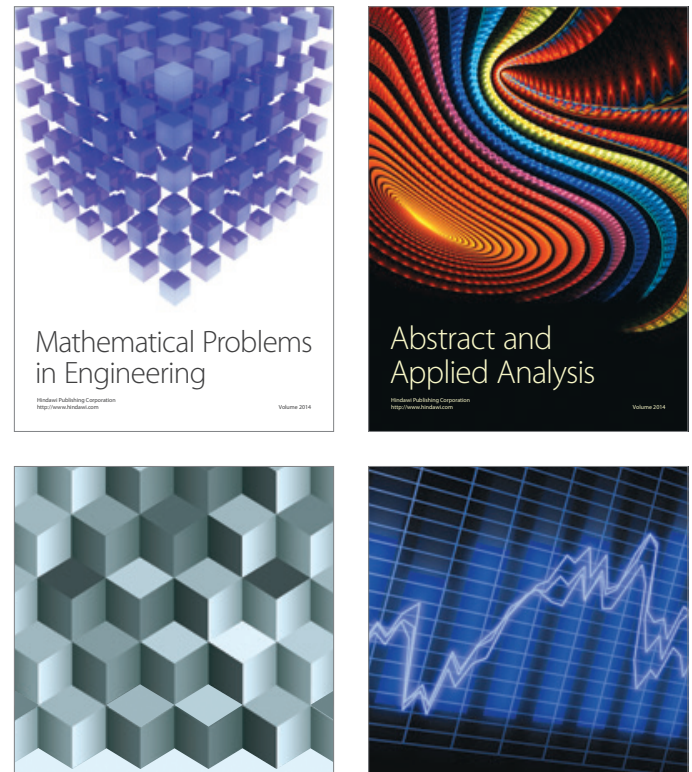

Journal of

Function Spaces

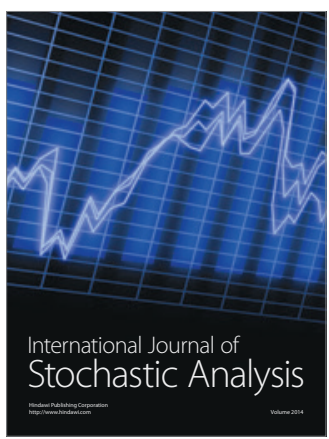

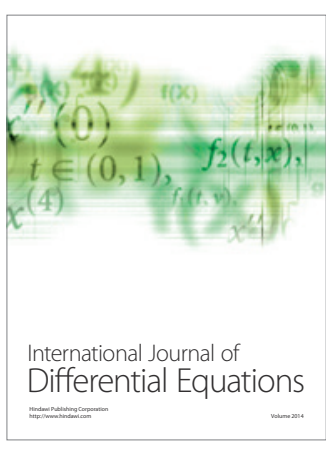
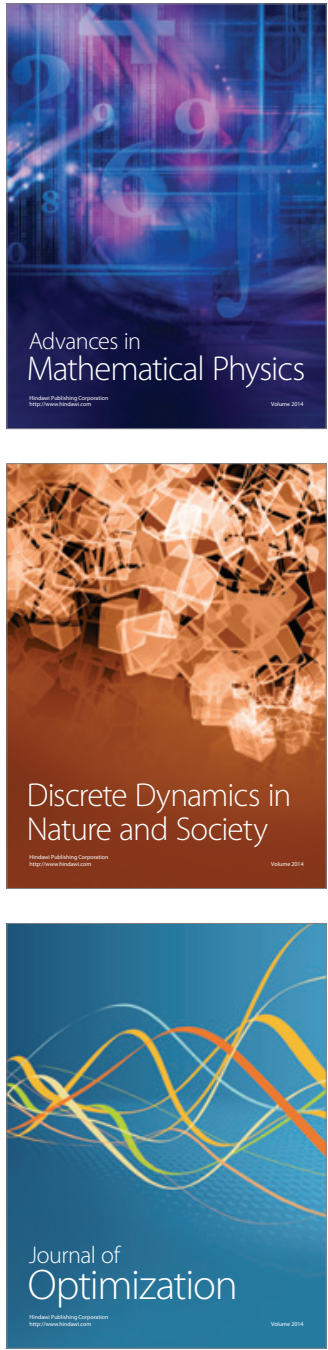